https://doi.org/10.46344/JBINO.2020.v09i06.11

\title{
DESMOCOLLIN 3 GENE (DSC3) EXPRESSION AND CANCER - IN SILICO EVALUATION
}

\author{
Chandreshwar Shukla, a N K Jain, a, Rajiv Modi, b Anand Khamar b, Bakulesh Khamar b* \\ a Gujarat University, Ahmedabad, Gujarat, India; \\ b Cadila Pharmaceuticals Limited, Ahmedabad, Gujarat, India
}

E-mail: bmk@cadilapharma.co.in

\begin{abstract}
Desmocollin-3 (DSC3) is DSC3 protein coding gene, expressed in some epithelial cancers. DSC3 expression has prognostic value in some cancers. Using cBioportal and KaplanMeier plotter DSC3 gene was evaluated in 12 cancers using TCGA data and compared with Hallmark gene expression. Cancers were grouped to have low (Group I), intermediate (Group II) and high (Group III) DSC3 expression. Higher DSC3 expression in group III is associated with better survival and in group I with poor survival. DSC3 expression correlated with desmosomal genes DSG3, DSC1, DSG1, PKP1 in spite of its known reciprocal relationship with DSC1, DSG1 and PKP1 under physiological conditions. Of 10 Hallmarks evaluated, three hallmarks had no correlation with DSC3. Activating invasion and metastasis (AIM) (genes SERPINB5, PERP, SNAI2) and Evading Growth Suppressors (EGS) (gene TP63) were the key hallmarks associated with DSC3 expression. Gene expression correlation with DSC3 across various hallmark genes reveals no correlation in group I cancers. Our finding demonstrate that higher DSC3 expression level may be useful for prognostication and needs further evaluation. The finding of this analysis also suggests critical role of TP63 in DSC3 expressing cancers and potentially a different biological process unique to DSC 3 expressing cancers.
\end{abstract}

Keywords: Cancer, DSC3 expression, Prognosis, Hallmark of cancer 


\section{Introduction}

Cancer arising from epithelial tissues (carcinoma) encompass $80-90 \%$ of all cancers (SEER Training Modules, 2020). In epithelia, the cells are adherent to each other via cadherin proteins located on lateral wall of epithelial cells (adherence junctions and desmosomes) to ensure tissue integrity and mechanical strength to withstand stress and strain (Gumbiner 1986). Cadherin proteins play an important role in bidirectional communication (through signalling pathways) between adjacent cells and surroundings (Klezovitch \& Vasioukhin 2015). They also regulate proliferation, polarity, migration and differentiation of epithelial cells and are responsible for formation, morphogenesis, maturation and homeostasis of epithelia. Epithelial cancers are known for abnormal expression of cadherin protein. E cadherin is widely studied and its loss is associated with epithelial mesenchymal transformation.

Desmocollin 3 (DSC3) is a calcium dependent desmosomal cadherin. It is the only cadherin which is p53 responsive (Cui et al., 2011) as well as tumour suppressive like(Cui et al., 2012). It is used as a diagnostic biomarker to differentiate squamous lung cancer and adenocarcinoma of lung (Tsuta et al., 2011 ). Expression of DSC3 protein is known in squamous non-small cell lung carcinoma (NSCLC), melanoma, ovarian cancer, bladder cancer, pancreatic cancer, esophageal cancer, cervical cancer, chondrosarcoma, oral cancer and colorectal cancer (Valentina et al., 2009, Khamar 2017, Salerno et al., 2016 ,
Uhlen et al., 2010, Uhlen et al., 2017, Robertson et al.,2017, Wang et al.,2014, Fitzgerald et al.,2011, Wang et al., 2011, Knösel et al.,2013). Its prognostic value is perplexing with better prognosis in squamous NSCLC (Belani et al.,2017, Cui et al.,2012) and poor prognosis in ovarian cancer.( Salerno et al., 2016).

Hallmark of cancers proposed by Hanahan and Weinberg organizes cancers with phenotypical and genotypical diversity into logical set of underlying organizing principles using knowledge about cell biology, histopathology, biochemistry, immunology, and pharmacology (Hanahan \& Weinberg 2000). Each hall mark is assigned with set of genes (Hanahan \& Weinberg 2011, lannuccelli et al., 2020). Information about genetic alteration in various cancers is available in The Cancer Genome Atlas (TCGA) database (Weinstein et al., 2013). Various tools have been developed to analyse The TCGA. cBioportal (Gao et al.,2013) and Kaplan Meier (K - M) plotter (Nagy et al.,2013) are two such tools. cBioportal is useful for assessment of gene expression, genetic alteration and gene correlation. The K-M plotter dataset is helpful for studying relationship between gene expression and survival in 12 cancers.

We undertook this study to evaluate for DSC3 gene expression in various cancer and, its relationship with survival, other desmosomal genes and hallmark genes using, cBioportal and K - M plotter tools.

\section{Methods}

Study characteristics: We have used cBioportal (cbioportal.org), and $K-M$ 
Plotter (kmplotter.org) data for studying DSC3 expression and its relationship with various parameters. A total of 12 cancer studies (Table S1), for which data is available in both datasets, were studied using as follows:

\section{cBioportal}

\section{DSC3 gene expression view of TCGA dataset:}

The level of DSC3 expression in tumour samples and change from normal tissue is analysed by using the Gene Expression Viewer tool [http://firebrowse.org/viewGene.html?ge ne=DSC3] (Fire Browse, Broad Institute of MIT and Harvard, 2019) which provides RNA-Seq by Expectation Maximization (RSEM) data for DSC3 in various cancer. The dataset filtered by selecting the type of cancer to view the RSEM data for DSC3 in cancer and in normal tissue (Gao et al., 2013). The esophageal carcinoma (ESCA) study included both adenocarcinoma and squamous cell carcinoma population, the RSEM data of individual population was extracted from cBioportal data set.

\section{DSC3 Gene alteration analysis:}

DSC3 RNA expressions are extracted from databases available at cBioPortal.org for Cancer Genomics. The query by gene "DSC3" was submitted in TCGA pancancer atlas datasets available (Table S1, 4974 human samples, from different cancer studies of TCGA Research Network (https://www.cbioportal.org/) (Gao et al., 2013). The 'cancer type summary' ribbon showed the alteration frequency of the gene in various cancers.

\section{Co-expression analysis:}

The 'Co-expression' ribbon for each cancer correlate the DSC3 mRNA expression with mRNA expression of other proteins. The spearman's correlation is determined for each sample by plotting DSC3 mRNA expression on $X$-axis and coexpressing gene on Y-axis. We have extracted the spearman's correlation for DSC3 with other desmosomal molecules and hallmarks of cancer (Hanahan \& Weinberg 2011, lannuccelli et al., 2020) for each cancer study. For gene correlation a spearman correlation value of $\geq 0.5$ was considered meaningful, and > 0.7 considered as highly correlated. (Table S4B). The TCGA data set of ESCA study include both adenocarcinoma (ESACA) and squamous cell carcinoma (ESSCA) population. RSEM data of individual population extracted for all the genes. The spearman correlation value with DSC 3 was calculated by RSEM data each gene using online statistical (https://www.socscistatistics.com/tests/sp earman/default2.aspx) (Jeremy Stangroom, Social Science Statistics, Updated 2018).

On basis of correlation values, the heat map and clustering of groups are assessed by

(http://heatmapper.ca/expression/) (Babicki et al., 2016), a web tool for visualizing clustering of multivariate data. The spearman's correlation values are uploaded to the tool and the Euclidean distance is measured on basis of average linkage in clustering method. The scale 
type is kept as none. The clustering is applied to both rows and column.

\section{K-M Plotter Survival analysis and Hazard Ratios estimations:}

The mRNA seq dataset of 'DSC3' gene symbol is analysed for survival assessment using auto select best cut-off option where all possible cut off values between the lower and upper quartiles are computed. Using auto select best cutoff option for DSC3, median overall survival (OS) was evaluated for all cancers. The Kaplan-Meier plot is drawn after choosing the cancer types (Nagy et al., 2018). The Log rank test is used as statistical inference between the two groups. $P$ value of $<0.05$ was considered statistically significant.

\section{Results}

\section{Study characteristics.}

DSC3 expression was evaluated using online platforms (cBioPortal and KMPlotter) in twelve (12) cancer types available on both. (Table 1 and S1)

\section{DSC3 expression in various cancer}

DSC3 expression level in twelve evaluated cancers vary in their median mRNA expression of from 1.5 in Kidney papillary cell carcinoma (KIPAN) to 19962 in ESSCA. Based on expression levels, cancers were segregated into 3 groups. cancer with low expression - KIPAN, Liver hepatocellular carcinoma (LIHC), Lung Adenocarcinoma (LUAD), Pancreatic adenocarcinoma (PAAD), ESACA, Ovarian serous cystadenocarcinoma (OV) (RSEM value:1 - 100; group I); intermediate expression - Breast invasive carcinoma (BRCA), Bladder urothelial carcinoma (BLCA) (RSEM value:100 1000; group II); and high expression - Lung Squamous cell carcinoma (LUSC) Cervical squamous cell carcinoma (CESC) Head and Neck Squamous cancer (HNSC) and ESSCA (RSEM value: >1000; group III). (Table 1).

\section{DSC3 expression in comparison to Normal}

KIPAN, LIHC, LUAD, ESACA, BRCA, and HNSC were found to have reduced expression of DSC3 (Normal/DSC3 < 1) compared to normal while ESSCA, LUSC and CESC had >10-fold increase in expression of DSC3 compared to normal. BLCA and PAAD also had increase in DSC3 expression by 1.6 fold and 3.8 fold respectively. This could not be analysed for OV as expression level in normal tissue was not available. (Table 1).

\section{Mutation levels of DSC3 TP53 and TP63:}

Number of patients harbouring DSC3 mutation ranged from $0.2 \%-4.2 \%$ of samples in various cancers. The mutational rate was highest for TP53 followed by $\mathrm{T} 63$ and DSC3 respectively (Table S2).

\section{DSC3 expression as prognostic marker for survival in various cancer (K-M plotter)}

K-M plotter identified cutoff vlue of DSC3 expression for each cancer separately based on survival and divided them in two cohort with DSC3 expresion higher or lower than cut off value. Comparison of cohorts with values higher or lower than cut off provided hazard ratio for each cancer separately. The cohort with DSC3 expression more than cut-off value had a poor survival in Group I and better 
survival in Group III. Cut-off value was also found to be highest in group III and lowest in group I. There was no correlation between mean, median and cut off values for DSC3 expression (Table 2).

\section{Correlation of DSC3 with other desmosomal components}

Amongst desmosomal components, significant correlation $(>0.5)$ with DSC3 was seen only in group II and group III (Table S3). Highest correlation of DSC3 was observed with DSG3 followed by DSC2, DSG1 and DSC1 respectively. Amongst armadillo proteins PKP1 was highly correlated followed by JUP and PKP3. Cluster analysis of these genes displayed difference in pattern of clustering across groups (Fig. 1). In group III, DSG2 and PKP2 were in one cluster while in group II, they were accompanied by DSG4, JUP, and PKP3. In group I, both DSG2, PKP2 were far distanced in different clusters. Similarly, DSG1 and PKP1 are clustered together in group II and III but not in group I. (Fig. 1).

\section{DSC3 co-relation with hallmarks of cancer}

Across 10 Hallmarks of cancer, 215 assigned genes to them were evaluated for correlation with DSC3 (Table 4A). Of 215 genes evaluated, 20 genes assigned to seven hallmarks were correlated (spearman correlation value $\geq 0.5$ ) with DSC3 (Table S4B). None of the genes in 3 hallmarks - Genome instability and mutation (GIM), Deregulating cellular energies (DCE) and Avoiding immune destruction (AID) correlated with DSC3
(Table S4B). Of remaining 7 hallmarks, significant correlation ( $\geq 25 \%$ of cancers) with DSC3 was seen in Activating invasion and metastasis (AIM; genes SERPINB5, PERP and SNAI2) and Evading growth Suppressors (EGS; gene TP63). No correlation between any hallmark and group I cancer was seen. Of the three genes belonging to AIM hallmark, SERPINB was correlated in all cancers of group II and III (Table 4SB), PERP correlated in group III and $\mathrm{CDH} 3$ in group II. A high correlation (Spearman value $>0.7$ ) of TP63 and PERP was observed in CESC, and SERPINB in BRCA.

Heatmap analysis of DSC3 correlated genes displayed difference in hierarchical clustering among the groups (Fig. 2). In group I, TP63 was clustered with SNAI2, followed by $\mathrm{CDH} 3$ (AIM) and SERPINB5. PERP belong to other cluster. In group II, PERP and TP63 were in same cluster closer to SNAI2, and $\mathrm{CDH} 3$ respectively. SERPINB5 also fall in same cluster but distanced from these gene groups. In group III, all the 3 genes (TP63, PERP, SERPINB5) were found nearest to each other as single group in positive cluster. SNAI2 belong to other cluster.

Negatively correlated genes formed a separate cluster in group II and III. Group I displayed mosaic clustering of these genes (Fig. 2). CDHI (AIM) found negative in group II, in place of PDIA6 (AIM) in group III, rest other genes were common in group II and III. 
Table 1: DSC3 mRNA levels in various tumour tissue

\begin{tabular}{|c|c|c|c|c|c|c|c|c|c|c|c|c|}
\hline \multicolumn{13}{|c|}{ DSC3 RNA Seq V2 } \\
\hline \multirow[b]{2}{*}{ Group } & \multirow[b]{2}{*}{ Cancer } & \multicolumn{4}{|c|}{ Tumor Tissue } & \multicolumn{4}{|c|}{ Normal Tissue } & \multicolumn{3}{|c|}{ Fold Change } \\
\hline & & Cases & Median & $\mathbf{1}^{\text {st }} \mathbf{Q}$ & $3^{\text {rd }} Q$ & Cases & Median & $1^{\text {st }} Q$ & $3^{\text {rd }} Q$ & Median & $\mathbf{1}^{\text {st }} \mathbf{Q}$ & $3^{\text {rd }} Q$ \\
\hline \multirow{6}{*}{$\begin{array}{l}\text { Group 1- } 0.1 \\
-100 \text { (Low } \\
\text { Expression; } \\
\text { L) }\end{array}$} & KIPAN & 720 & 1.5 & 0.7 & 3.6 & 121 & 2.3 & 1.2 & 4.2 & 0.64 & 0.6 & 0.9 \\
\hline & LIHC & 231 & 2.0 & 0.9 & 6.1 & 42 & 3.9 & 2.0 & 8.5 & 0.52 & 0.5 & 0.7 \\
\hline & LUAD & 496 & 10 & 3.6 & 38 & 54 & 18.1 & 5.1 & 37.5 & 0.56 & 0.7 & 1.0 \\
\hline & PAAD & 176 & 46 & 17 & 111 & 4 & 12.0 & 8.9 & 16.1 & 3.80 & 1.9 & 6.9 \\
\hline & *ESACA & 87 & 68 & 14 & 493 & 10 & 202 & 44 & 976 & 0.07 & 0.3 & 0.5 \\
\hline & $\mathrm{OV}$ & 307 & 86 & 29 & 223 & NA & NA & NA & NA & - & - & - \\
\hline \multirow{3}{*}{$\begin{array}{l}\text { Group 2-100 } \\
-\quad 1000 \\
\text { (Intermediate } \\
\text { Expression; } \\
\text { I) }\end{array}$} & BRCA & 1090 & 120 & 22 & 455 & 111 & 1097 & 690 & 1663 & 0.11 & 0.0 & 0.3 \\
\hline & & & & & & & & & & & & \\
\hline & BLCA & 394 & 639 & 29 & 3566 & 18 & 402 & 260 & 1176 & 1.59 & 0.1 & 3.0 \\
\hline \multirow{4}{*}{$\begin{array}{l}\text { Group 3- } \\
>1000 \text { (High } \\
\text { Expression; } \\
\text { H) }\end{array}$} & LUSC & 501 & 6208 & 2896 & 10086 & 51 & 33 & 16 & 76 & 195 & 184.8 & 133.4 \\
\hline & CESC & 306 & 10086 & 495 & 18820 & 3 & 48 & 23 & 75 & 209 & 21.7 & 250.7 \\
\hline & HNSC & 522 & 15287 & 10086 & 23170 & 44 & 16384 & 7643 & 21619 & 0.93 & 1.3 & 1.1 \\
\hline & *ESSCA & 95 & 19962 & 12505 & 28423 & 10 & 202 & 44 & 976 & 98.8 & 284.2 & 29.1 \\
\hline
\end{tabular}

* Normal sample expression from esophageal carcinoma (ESCA) was used for both ESACA and ESSCA. 
Table 2: DSC3 expression as prognostic marker for survival in various cancer (K-M plotter)

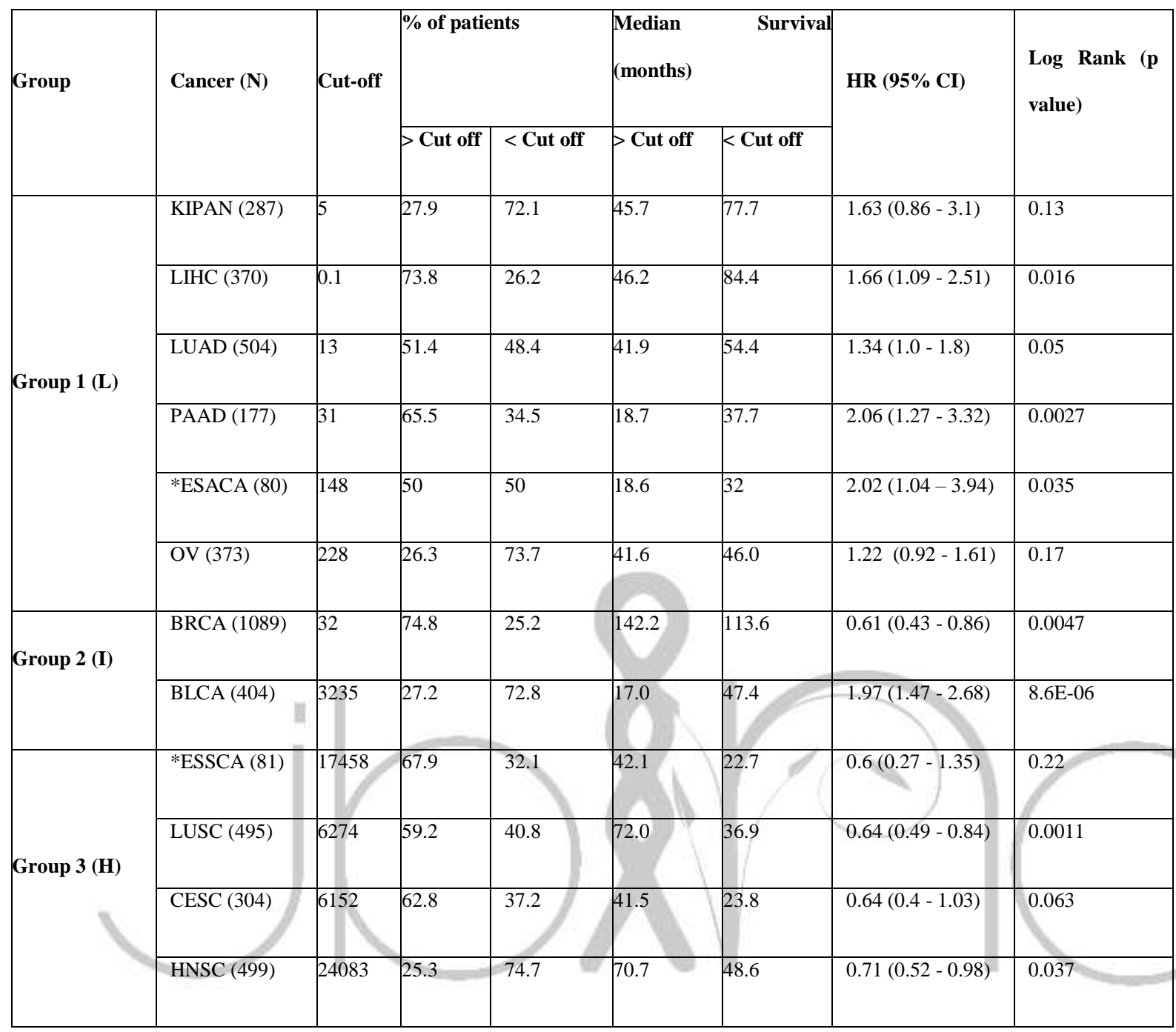

* Expression range is extracted from K-M plotter data set and grouped on basis of cut-off value. 
Fig. 1: Cluster analysis of spearman correlation of DSC3 with Desmosomal molecules.

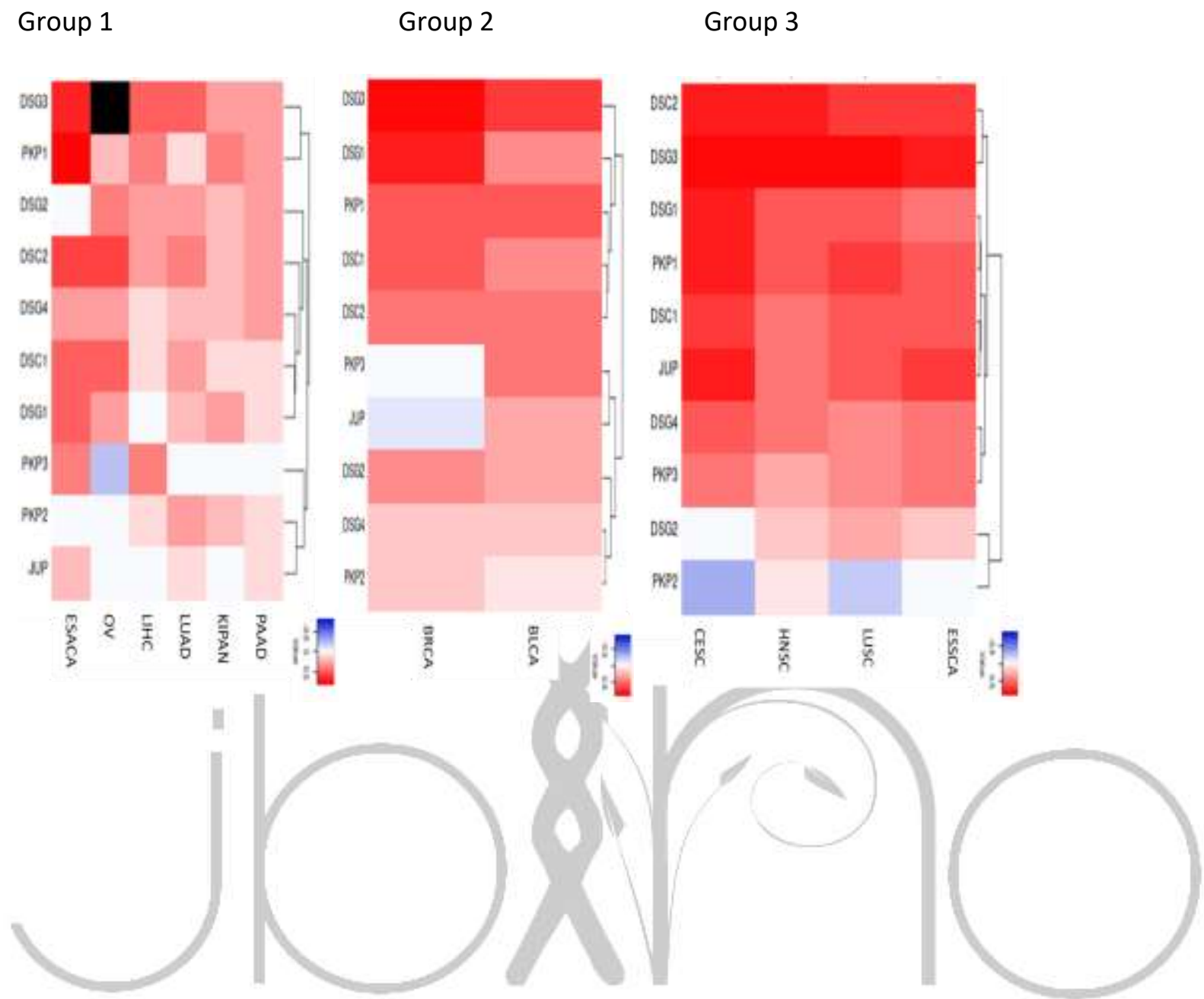


Fig. 2: Cluster analysis of spearman correlation $(\geq 0.5)$ of DSC3 with hallmarks of cancer molecules.

\section{Group 1}
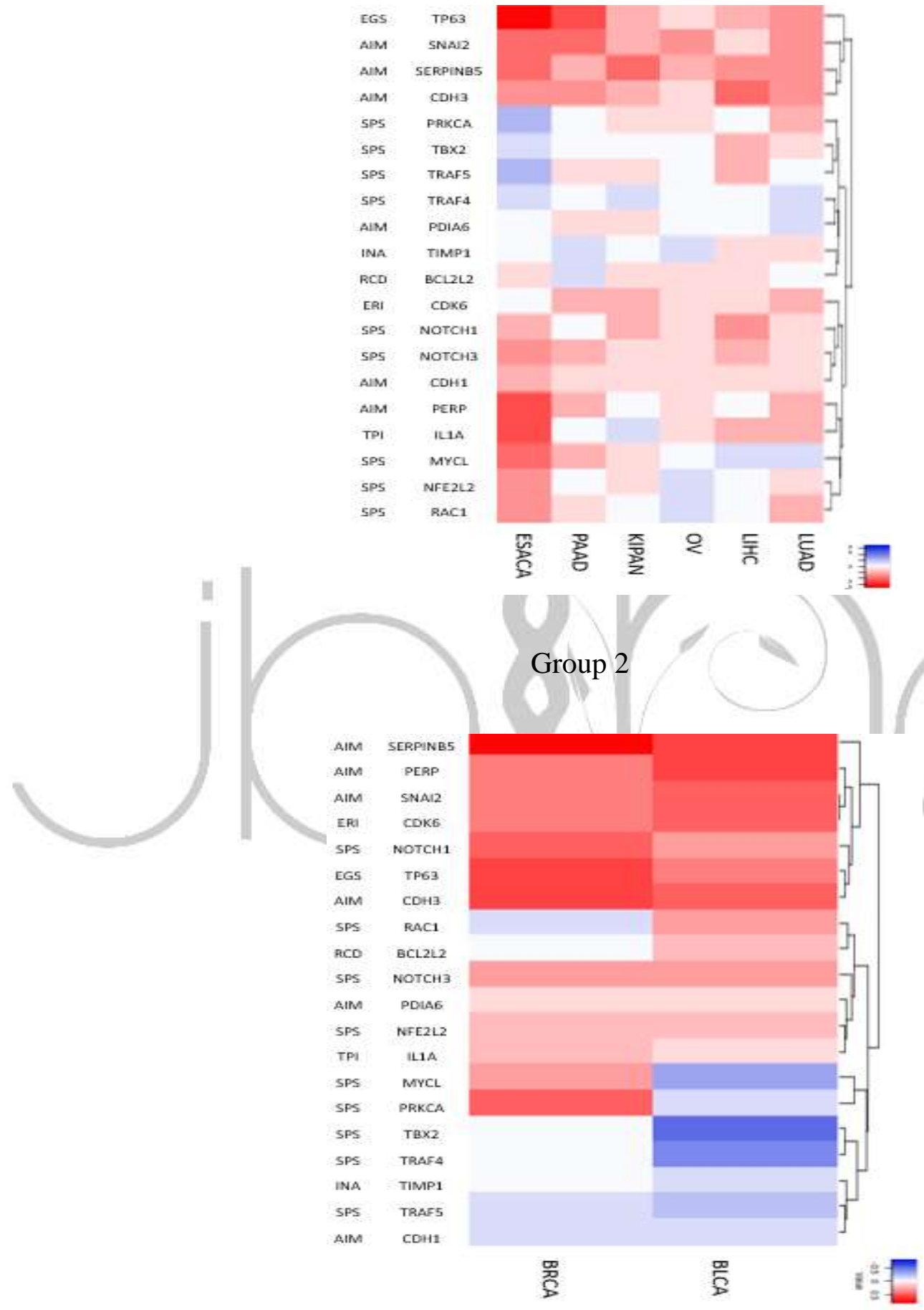


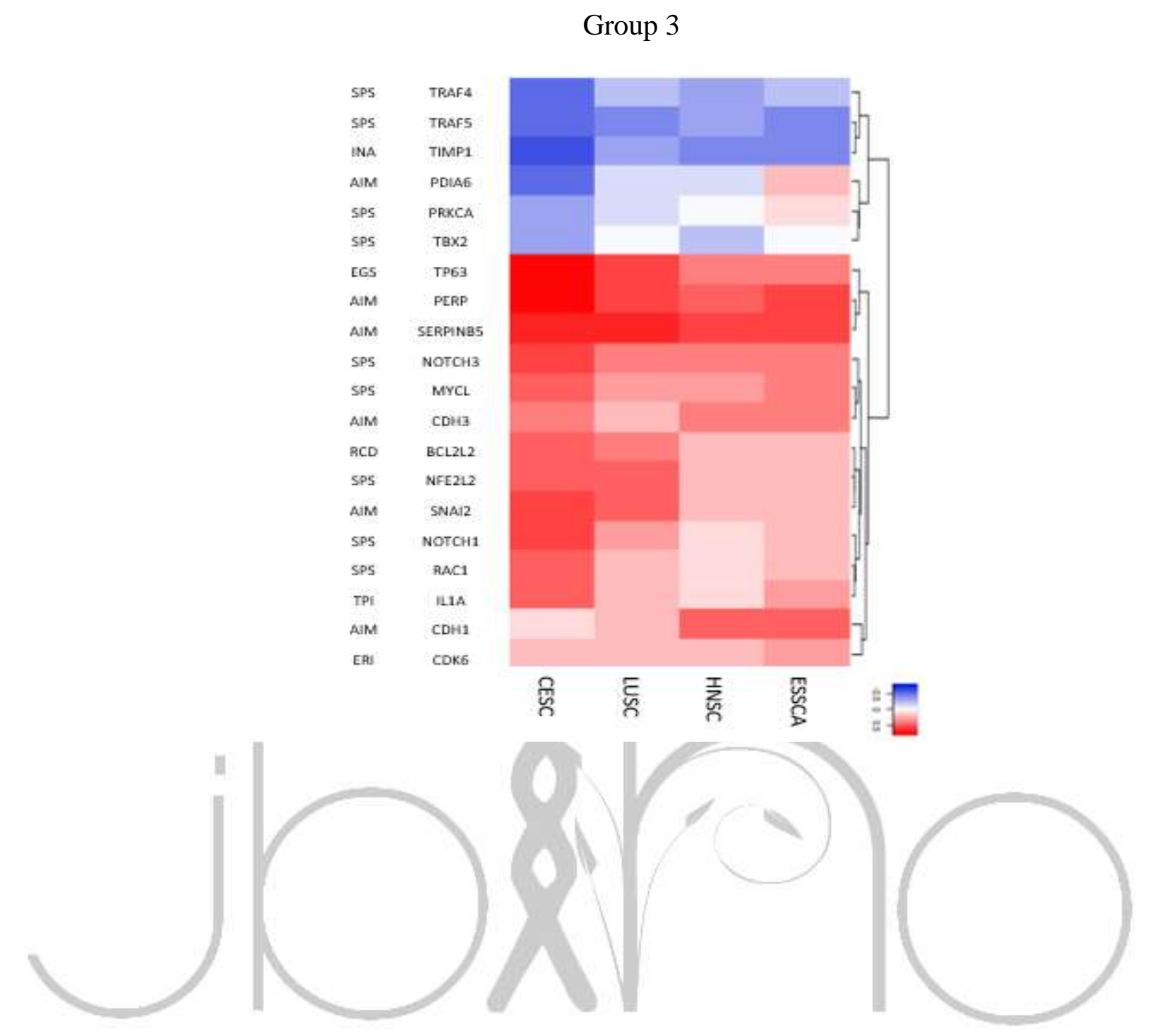




\section{Supplementary Appendix:}

This appendix has been provided by the authors to give readers additional information about their work.

\section{Table of Contents}

Table S1: Cancer studies analysed in this article 11

Table S2: DSC3 structural alteration in various cancer 12

Table S3: DSC3 co-relation with other desmosomal components. 13

Table S4A: Spearman correlation analysis with cancer hallmark genes .14

Table S4B: Correlated cancer hallmark genes (spearman value $\geq 0.5$ ) with DSC3 16

Table S1: Cancer studies analysed in this article

\begin{tabular}{|c|c|c|c|c|}
\hline TCGA PanCancer Atlas & Initial & $\begin{array}{l}\text { Sample No. } \\
\text {-Gene } \\
\text { expression } \\
\text { viewer }\end{array}$ & $\begin{array}{l}\text { Sample No. } \\
\text { - cBioPortal }\end{array}$ & $\begin{array}{l}\text { Sample No. } \\
\text {-K-M } \\
\text { Plotter }\end{array}$ \\
\hline Kidney renal papillary cell carcinoma & KIPAN & 720 & 283 & 287 \\
\hline Liver hepatocellular carcinoma & LIHC & 231 & 372 & 370 \\
\hline Lung adenocarcinoma & LUAD & 496 & 566 & 504 \\
\hline Pancreatic adenocarcinoma & PAAD & 176 & 184 & 177 \\
\hline Esophageal adenocarcinoma & ESACA & - & 87 & 80 \\
\hline Ovarian serous cystadenocarcinoma & $\mathrm{OV}$ & 307 & 585 & 373 \\
\hline Breast invasive carcinoma & BRCA & 1090 & 1084 & 1089 \\
\hline Bladder urothelial carcinoma & BLCA & 394 & 411 & 404 \\
\hline Lung Squamous cell carcinoma & LUSC & 501 & 487 & 495 \\
\hline Cervical squamous cell carcinoma & CESC & 306 & 297 & 304 \\
\hline Head \& Neck Squamous cancer & HNSC & 522 & 523 & 499 \\
\hline Esophageal Squamous carcinoma & ESSCA & - & 95 & 81 \\
\hline
\end{tabular}


Table S2: DSC3 structural alteration in various cancer

\begin{tabular}{|c|c|c|c|c|c|c|c|c|c|c|c|c|c|}
\hline \multirow{2}{*}{ Cancer } & \multirow{2}{*}{$\begin{array}{l}\text { Sample } \\
\text { No. }\end{array}$} & \multicolumn{3}{|c|}{ Mutation } & \multicolumn{3}{|c|}{ Deep Deletion } & \multicolumn{3}{|c|}{ Amplification } & \multicolumn{3}{|c|}{ Multiple Alterations } \\
\hline & & DSC3 & TP63 & TP53 & DSC3 & TP63 & TP53 & DSC3 & TP63 & TP53 & DSC3 & TP63 & TP53 \\
\hline KIPAN & 283 & 1.1 & 0.4 & 3.2 & 0.4 & NR & 0.7 & NR & 0.7 & NR & NR & NR & $\mathrm{NR}$ \\
\hline LIHC & 372 & 0.3 & 0.8 & 28.5 & NR & NR & 1.3 & 0.3 & 0.8 & 0.3 & NR & 0.3 & 1.1 \\
\hline LUAD & 566 & 4.2 & 2.3 & 51.8 & 0.2 & 0.7 & 0.2 & 0.5 & 1.6 & 0.2 & NR & 0.2 & 1.4 \\
\hline PAAD & 184 & 0.5 & 0.5 & 54.4 & 1.6 & NR & 0.5 & 3.8 & 1.6 & 1.1 & NR & NR & 4.4 \\
\hline ESACA & 87 & 1.2 & NR & 79.3 & 2.3 & NR & NR & 2.3 & 5.8 & NR & NR & NR & 1.2 \\
\hline OV & 585 & 0.3 & 0.5 & 60.6 & 0.5 & 0.2 & 0.3 & 3.6 & 15.4 & 1.9 & NR & 0.2 & 3.3 \\
\hline BRCA & 1084 & 0.7 & 0.4 & 31.6 & NR & 0.1 & 0.7 & 0.7 & 1.6 & 0.2 & NR & NR & 0.9 \\
\hline BLCA & 411 & 2.7 & 3.4 & 48.4 & 0.2 & NR & 1.5 & 1.2 & 2.9 & 1.0 & $\mathrm{NR}$ & 0.5 & 1.0 \\
\hline LUSC & 487 & 1.0 & 2.7 & 8.4 & NR & NR & 1.0 & NR & 15.8 & NR & NR & 0.3 & NR \\
\hline CESC & 297 & 0.2 & 1.5 & 66.7 & 0.4 & NR & NR & 0.8 & 15.1 & NR & NR & 0.8 & 1.7 \\
\hline HNSC & 523 & NR & NR & 91.6 & 2.1 & NR & 1.1 & 3.2 & 31.6 & NR & NR & 2.1 & 1.1 \\
\hline ESSCA & 95 & 1.1 & 0.4 & 3.2 & 0.4 & NR & 0.7 & NR & 0.7 & NR & NR & NR & NR \\
\hline
\end{tabular}


Table S3: DSC3 co-relation with other desmosomal components

\begin{tabular}{|c|c|c|c|c|c|c|c|c|c|c|}
\hline Cancer & DSC1 & DSC2 & DSG1 & DSG2 & DSG3 & DSG4 & PKP1 & PKP2 & PKP3 & JUP \\
\hline KIPAN & 0.1 & 0.2 & 0.3 & 0.2 & 0.3 & 0.2 & 0.4 & 0.2 & 0.0 & 0.0 \\
\hline LIHC & 0.1 & 0.3 & 0.0 & 0.3 & 0.5 & 0.1 & 0.4 & 0.1 & 0.4 & 0.0 \\
\hline LUAD & 0.3 & 0.4 & 0.2 & 0.3 & 0.5 & 0.2 & 0.1 & 0.3 & 0.0 & 0.1 \\
\hline PAAD & 0.1 & 0.3 & 0.1 & 0.3 & 0.3 & 0.3 & 0.3 & 0.1 & 0.0 & 0.1 \\
\hline ESACA & 0.5 & 0.6 & 0.5 & 0.0 & 0.7 & 0.3 & 0.8 & 0.0 & 0.4 & 0.2 \\
\hline $\mathrm{OV}$ & 0.5 & 0.6 & 0.3 & 0.4 & NA & 0.3 & 0.2 & 0.0 & -0.2 & 0.0 \\
\hline BRCA & 0.6 & 0.5 & 0.8 & 0.4 & 0.9 & 0.2 & 0.6 & 0.2 & 0.0 & -0.1 \\
\hline BLCA & 0.4 & 0.5 & 0.4 & 0.3 & 0.7 & 0.2 & 0.6 & 0.1 & 0.5 & 0.3 \\
\hline LUSC & 0.6 & 0.7 & 0.6 & 0.3 & 0.9 & 0.4 & 0.7 & -0.2 & 0.4 & 0.6 \\
\hline CESC & 0.7 & 0.8 & 0.8 & 0.0 & 0.9 & 0.6 & 0.8 & -0.3 & 0.5 & 0.8 \\
\hline HNSC & 0.5 & 0.8 & 0.6 & 0.2 & 0.9 & 0.5 & 0.6 & 0.1 & 0.3 & 0.5 \\
\hline ESSCA & 0.6 & 0.7 & 0.5 & 0.2 & 0.8 & 0.5 & 0.6 & 0.0 & 0.5 & 0.7 \\
\hline
\end{tabular}


Table S4A: Spearman correlation analysis with cancer hallmark genes

\begin{tabular}{|c|c|c|c|c|c|c|c|c|c|}
\hline $\begin{array}{l}\text { Sustained } \\
\text { proliferative } \\
\text { signalling } \\
\text { (SPS) }\end{array}$ & $\begin{array}{l}\text { Evading } \\
\text { growth } \\
\text { Suppressors } \\
\text { (EGS) }\end{array}$ & $\begin{array}{l}\text { Activating } \\
\text { invasion and } \\
\text { metastasis } \\
\text { (AIM) }\end{array}$ & $\begin{array}{l}\text { Enabling } \\
\text { Replicative } \\
\text { immortality } \\
\text { (ERI) }\end{array}$ & $\begin{array}{l}\text { Inducing } \\
\text { angiogenesis } \\
\text { (INA) }\end{array}$ & $\begin{array}{l}\text { Resisting } \\
\text { cell } \\
\text { death } \\
\text { (RCD) }\end{array}$ & $\begin{array}{l}\text { Avoiding } \\
\text { immune } \\
\text { destruction } \\
\text { (AID) }\end{array}$ & $\begin{array}{l}\text { Tumour } \\
\text { promoting } \\
\text { Inflammation } \\
\text { (TPI) }\end{array}$ & $\begin{array}{l}\text { Genome } \\
\text { instability } \\
\text { and } \\
\text { mutation } \\
\text { (GIM) }\end{array}$ & $\begin{array}{l}\text { Deregulating } \\
\text { cellular } \\
\text { energies } \\
\text { (DCE) }\end{array}$ \\
\hline AP1B1 & TP53 & CRABP2 & RANGAP1 & AGTR2 & BAK1 & ARG1 & CD40 & BRCA1 & BRAF \\
\hline ASXL1 & TP63 & BACH1 & MCM5 & ANG & BAX & CCR7 & IL1A & BRCA2 & HIF1A \\
\hline BAD & TP73 & PTTG1 & MCM2 & CD36 & BCL2 & CD27 & IL1R1 & ERCC1 & HIF1AN \\
\hline BAP1 & MDM2 & WASF3 & CCND1 & COL18A1 & BCL2L1 & CD274 & IRAK3 & ERCC2 & HIF3A \\
\hline BCOR & CDK2 & CCL5 & CDT1 & CSF3 & BCL2L2 & CD276 & NR3C1 & ERCC3 & IDH1 \\
\hline CAMTA1 & RB1 & CSF1 & CDK6 & CXCL12 & BECN1 & CD80 & TNF & ERCC4 & IDH2 \\
\hline CCNA1 & STK11 & PDIA6 & CDK4 & CXCL8 & CASP3 & CD86 & & ERCC5 & KRAS \\
\hline CCND1 & NF2 & EZR & DAXX & CXCR4 & CASP8 & CXCL9 & & ERCC6 & LDHA \\
\hline CDKN2A & TGFB1 & PERP & ATRX & FGF2 & FOXA1 & CXCR6 & & ERCC8 & PFKFB2 \\
\hline CDKN2B & TGFB2 & SERPINB5 & CCND2 & MMP1 & FOXL2 & CYBB & & HRNR & POGLUT1 \\
\hline CTNNB1 & TGFB3 & CDH1 & AURKA & PDGFA & FOXO1 & IDO1 & & MLH1 & PRKAA1 \\
\hline GSTA1 & & $\mathrm{CDH} 2$ & CCND3 & TGFA & FOXO3 & IL12B & & MRE11 & PRKAA2 \\
\hline HHEX & & $\mathrm{CDH} 3$ & CDKN2A & THBS1 & FOXO4 & IL1B & 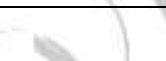 & MSH2 & \\
\hline HMOX1 & & SNAI1 & TERT & TIMP1 & HIP1 & IL27 & & PMS1 & \\
\hline JUN & & SNAI2 & & TIMP2 & HLF & IL4 & $=$ & PMS2 & \\
\hline LEF1 & & TWIST1 & & TIMP3 & MEIS1 & IL6 & & PMS2 & \\
\hline LRP1B & & ZEB1 & & TIMP4 & NAE1 & LAG3 & & POLH & \\
\hline MAPK1 & Wen & int & 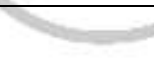 & VEGFA & NFIL3 & MARCO & & RAD50 & 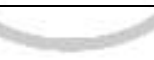 \\
\hline MEIS1 & & & & VEGFB & NFKB1 & NKG7 & & RAD52 & \\
\hline MGLL & & & & VEGFC & NFKB2 & NOS2 & & RECQL4 & \\
\hline MLLT11 & & & & VEGFD & NODAL & PDCD1LG2 & & WRN & \\
\hline MN1 & & & & & PLAG1 & PPARG & & XPA & \\
\hline MTOR & & & & & PML & SOCS3 & & & \\
\hline MYC & & & & & STAT5A & STAT1 & & & \\
\hline$\overline{\text { MYCL }}$ & & & & & TRAF7 & STAT6 & & & \\
\hline NFE2L2 & & & & & TRIM27 & $\begin{array}{l}\text { TIGIT } \\
\end{array}$ & & & \\
\hline NFKB1 & & & & & WWTR1 & & & & \\
\hline \multicolumn{10}{|l|}{ NODAL } \\
\hline \multicolumn{10}{|l|}{ NOTCH1 } \\
\hline \multicolumn{10}{|l|}{ NOTCH2 } \\
\hline \multicolumn{10}{|l|}{ NOTCH3 } \\
\hline \multicolumn{10}{|l|}{ NOTCH4 } \\
\hline \multicolumn{10}{|l|}{ NQO1 } \\
\hline NR4A3 & & & & & & & & & \\
\hline
\end{tabular}




\begin{tabular}{|l|l|l|l|l|l|l|l|l|l|}
\hline PIK3CA & & & & & & & & & \\
\hline
\end{tabular}


Table S4B: Correlated cancer hallmark genes (spearman value $\geq 0.5$ ) with DSC3

\begin{tabular}{|c|c|c|c|c|c|c|c|c|c|c|c|c|c|}
\hline Hallmark & Correlated Gene & KIPAN & LIHC & LUAD & PAAD & ESACA & OV & BRCA & BLCA & LUSC & CESC & HNSC & ESSCA \\
\hline SPS & MYCL & 0.1 & -0.1 & -0.1 & 0.2 & 0.4 & 0.0 & 0.3 & -0.3 & 0.3 & 0.5 & 0.3 & 0.4 \\
\hline SPS & NFE2L2 & 0.1 & 0.0 & 0.1 & 0.0 & 0.3 & -0.1 & 0.2 & 0.2 & 0.5 & 0.5 & 0.2 & 0.2 \\
\hline SPS & NOTCH1 & 0.2 & 0.3 & 0.1 & 0.0 & 0.2 & 0.1 & 0.5 & 0.3 & 0.3 & 0.6 & 0.1 & 0.2 \\
\hline SPS & NOTCH3 & 0.1 & 0.2 & 0.1 & 0.2 & 0.3 & 0.1 & 0.3 & 0.3 & 0.4 & 0.6 & 0.4 & 0.4 \\
\hline SPS & RAC1 & 0.0 & 0.0 & 0.2 & 0.1 & 0.3 & -0.1 & -0.1 & 0.3 & 0.2 & 0.5 & 0.1 & 0.2 \\
\hline SPS & PRKCA & 0.1 & 0.0 & 0.2 & 0.0 & -0.2 & 0.1 & 0.5 & -0.1 & -0.1 & -0.3 & 0.0 & 0.1 \\
\hline SPS & TBX2 & 0.0 & 0.2 & 0.1 & 0.0 & -0.1 & 0.0 & 0.0 & -0.5 & 0.0 & -0.3 & -0.2 & 0.0 \\
\hline SPS & TRAF4 & -0.1 & 0.0 & -0.1 & 0.0 & -0.1 & 0.0 & 0.0 & -0.4 & -0.2 & -0.5 & -0.3 & -0.2 \\
\hline SPS & TRAF5 & 0.1 & 0.2 & 0.0 & 0.1 & -0.2 & 0.0 & -0.1 & -0.2 & -0.4 & -0.5 & -0.3 & -0.4 \\
\hline EGS & TP63 & 0.2 & 0.2 & 0.3 & 0.5 & 0.7 & 0.1 & 0.6 & 0.4 & 0.6 & 0.8 & 0.4 & 0.4 \\
\hline AIM & PDIA6 & 0.1 & 0.0 & -0.1 & 0.1 & 0.0 & 0.0 & 0.1 & 0.1 & -0.1 & -0.5 & -0.1 & 0.2 \\
\hline AIM & PERP & 0.0 & 0.0 & 0.2 & 0.2 & 0.5 & 0.1 & 0.4 & 0.6 & 0.6 & 0.8 & 0.5 & 0.6 \\
\hline AIM & SERPINB5 & 0.4 & 0.3 & 0.3 & 0.2 & 0.4 & 0.2 & 0.8 & 0.6 & 0.7 & 0.7 & 0.6 & 0.6 \\
\hline AIM & CDH1 & 0.1 & 0.1 & 0.1 & 0.1 & 0.2 & 0.1 & -0.1 & -0.1 & 0.2 & 0.1 & 0.5 & 0.5 \\
\hline AIM & $\mathrm{CDH} 3$ & 0.2 & 0.4 & 0.3 & 0.3 & 0.3 & 0.1 & 0.6 & 0.5 & 0.2 & 0.4 & 0.4 & 0.4 \\
\hline AIM & SNAI2 & 0.2 & 0.1 & 0.3 & 0.4 & 0.4 & 0.3 & 0.4 & 0.5 & 0.5 & 0.6 & $0.2=$ & 0.2 \\
\hline ERI & CDK6 & 0.2 & 0.1 & 0.2 & 0.2 & 0.0 & 0.1 & 0.4 & 0.5 & 0.2 & 0.2 & 0.2 & 0.3 \\
\hline INA & TIMP1 & 0.0 & 0.1 & 0.1 & -0.1 & 0.0 & -0.1 & 0.0 & -0.1 & -0.3 & -0.6 & -0.4 & -0.4 \\
\hline RCD & BCL2L2 & 0.1 & 0.1 & 0.0 & -0.1 & 0.1 & 0.1 & 0.0 & 0.2 & 0.4 & 0.5 & 0.2 & 0.2 \\
\hline TPI & IL1A & -0.1 & 0.2 & 0.2 & 0.0 & 0.5 & 0.1 & 0.2 & 0.1 & 0.2 & 0.5 & 0.1 & 0.3 \\
\hline
\end{tabular}




\section{Discussion:}

Higher than normal expression of DSC3 seen in pancreatic, bladder, squamous esophageal, cervical squamous cancer and squamous lung cancer (Table 1) correlates with DSC3 protein seen in these patients (Valentina et al.,2009, Uhlen et al., 2010, Uhlen et al., 2017, Robertson et al.,2017, Wang et al.,2014,). Epigenetic silencing can be the reason for discordance between molecular expression and protein expression as seen in breast cancer (Oshiro et al., 2005).

Mutations of p53 are seen in majority of cancers and is a known major driver mutation (Bailey et al.,2018). In spite of being p53 dependent gene, DSC3 gene mutations were very low across various cancers studied indicating presence of different regulatory mechanism.

Better survival is seen in cancers with high DSC3expression (Group-III) and poor survival in low DSC3 expression (Group-I) (Table 2) in this study is in line with previous findings of better survival in LUSC (high DSC3 expression; Group III) (Belani et al.,2017, Cui et al.,2012) and poor survival in ovarian cancer (low DSC3 expression ; Group I) (Salerno et al., 2016). The correlation of survival with DSC3 expression level seen this study may explain the difference seen LUSC and ovarian cancer, and also suggest to consider DSC3 expression while evaluating DSC3 as a prognostic parameter in future studies.

Desmosomal integrity is prognostic of better outcome (Jodi et al.,2014). No correlation was seen between DSC3 expression level and desmosomal components in group I cancers and variable correlation was seen between DSC3 and desmosomal component seen in group II and III cancers. This is indicative of loss of desmosome integrity in this group I and may have contributed to poor survival seen in this group while using DSC3 as a prognostic parameter. High correlation of DSC3 with DSG3 (Fig. 1) in group II and III of this study could be due to their adjacent location on chromosome 18 (Berika \& Garrod 2014). Correlation of DSC1, DSG1, PKP1 with DSC3 seen in this study is a novel finding (Fig. 1, Table S3). DSC3 as well as DSG3 expression is described to have reciprocal relationship with DSC1, DSG 1 and $\mathrm{PKP1}$ in normal as well as cancers (Jodi et al.,2014, Berika \& Garrod 2014, Ferone et al.,2013, Khan et al.,2006, Green etal.,2019, Dusek \& Attardi 2011). Initially DSG 1 and DSC 1 are expressed in basal layers and then migrate to superficial layers (Berika \& Garrod 2014, Ferone et al.,2013, Khan et al.,2006, Green etal.,2019). It appears that they are retained in basal proliferative layers in along with DSC3 in group II and III cancers. PKP1 is known for growth control and hyper adhesion (Ferone et al.,2013, Khan et al.,2006, Green etal.,2019, Dusek \& Attardi 2011). Correlation of PKP1 with DSC3 in this group might have contributed to better survival.

DSC3 expression was found to be correlated with various genes representing various hallmark in Group ॥ and III (higher DSC3) and with none in group I. (Fig. 2, Table S4B). Correlation between DSC3 and Group II and III cancer was seen for EGS and AIM hallmark. Genes maximally correlated 
with DSC3 across both groups are SERPINB5 (AIM), PERP (AIM), TP63 (EGS), and SNAI2 (AIM) (Fig. 2, Table S4B). Like DSC3, SERPINB5 and PERP are desmosomal proteins and their correlation with DSC3 suggest desmosomal integrity (Dusek \& Attardi $2011)$. DSC3, PERP, SERPINB5, and SNAI2 gene expression is known to be TP63 dependent (Ferone et al.,2013, Khan et al.,2006). DSC1, DSG1, DSG3 are other desmosomal genes which are TP63 regulated and are correlated with DSC3 expression in group II and III. Increase in p63 is reported in squamous cancer of lung, head - neck, esophagel cancer, cervical cancer, bladder cancer (Moses et al.,2019). This indicate the significance of TP63 in DSC3 expressing cancers. DSC3 is a known master regulator as well as tumour suppressor (Moses et al., 2019, Ihrie et al., 2005).

Of TP63 isoforms, $\triangle N P 63$ is described to be associated with DSC3 in various disorder (Koster et al., 2014). $\triangle \mathrm{NP} 63$ is also known oncogene which promotes cancer development but controls invasion and metastasis (Srivastava et al.,2018). DSC3, PKP1, Maspin and PERP (Green et al.,2019) are known for tumour suppression and inhibition of tumour invasion and metastasis (Jodi et al.,2014, Dzinic et al., 2015, Bodenstine et al.,2012, Lockett et al.,2005). All these factors might have contributed to correlation between DSC3 expression and better survival.

Epithelial mesenchymal transition (EMT) is associated with appearance of $\mathrm{CDH} 3$ and disappearance of CDH1, (Dusek et al., 2011). both belonging to AIM hallmark as well as adherence junction. Like CDH3, SNAI2 (AIM) is also associated with EMT and also has a reciprocal relationship with $\mathrm{CDH} 1$. In this study correlation of DSC3 with CDH1 expression and SNAI2 expression were mutually exclusive. HNSC and ESSCA expressed CDHI and CESC, LUSC expressed SANI2. Relationship between $\mathrm{CDH} 1$ and desmosomal proteins is well known (Dusek et al., 2011). but the correlation of mesenchymal marker CDH3 and SNAI2 with DSC3 an epithelial marker seems to be unique and is indicative of proposed EM3 stage within EMT spectrum (Nieto et al.,2016) in group I and II.

Present study suggests, DSC3 expression level may be useful for prognostication. It also suggests critical role of TP63 regulated desmosomal components in DSC3 expressing cancers. Association of DSG 1, DSC 1, PKP1, and SNAI2 with DSC3 in group II and III cancers is indicative of different biological process in these cancers. This study utilised TCGA dataset for DSC3 gene expression and its correlation. In all cancers studied, no information about epigenetic changes and DSC3 protein expression was available and so could not be studied. Epigenetic silencing of DSC3 gene is well known (Babicki et al., 2016, J. Pan et al., 2014). This needs to be evaluated for better prognostication in future studies. Correlation between DSC 1, DSG1, PKP1 and DSC3 seen in this study is indicative of different biological process which needs further exploration.

\section{Declarations:}

Funding: Not applicable 
Conflicts of interest/Competing interests: The authors declare no conflict of interest.

Ethics approval: Not applicable

\section{Reference}

1. SEER Training Modules, Module Name. U. S. National Institutes of Health, National Cancer Institute (2020).

https://training.seer.cancer.gov/dis ease/categories/classification.html. Accessed 03 Feb 2020.

2. B.M. Gumbiner, Cell Adhesion: The Molecular Basis of Tissue Architecture and Morphogenesis. Cell 84 (1996), 345-357.

3. O. Klezovitch and V. Vasioukhin, Cadherin signaling: keeping cells in touch [version 1; peer review: 3 approved]. F1000Research 4(F1000 Faculty Rev) (2015) 550

4. T. Cui, Y. Chen, L. Yang, T. Knösel, K. Zöller, O. Huber, I. Petersen, DSC3 expression is regulated by $\mathrm{p} 53$, and methylation of DSC3 DNA is a prognostic marker in human colorectal cancer. Br J Cancer 104 (2011),1013-1019.

5. T. Cui, Y. Chen, L. Yang, T. Knösel, O. Huber, Pacyna-Gengelbach, I. Petersen, The p53 target gene desmocollin 3 acts as a novel tumor suppressor through inhibiting EGFR/ERK pathway in human lung cancer, Carcinogenesis 33 (2012), 2326-2333.

6. K. Tsuta, Y. Tanabe, A. Yoshida, F. Takahashi, A.M. Maeshima, H.
Asamura, H. Tsuda, Utility of 10 Immunohistochemical Markers Including Novel Markers (Desmocollin-3, Glypican 3, S100A2, S100A7, and Sox-2) for Differential Diagnosis of Squamous Cell Carcinoma from Adenocarcinoma of the Lung, Journal of Thoracic Oncology 6 (2011), 1190-1199.

7. M. Valentina, C. Paolo, R. Luisella, T. Veronica, V. Marco, P. Giuseppe, V.S. Giorgio, P. Mauro, Desmocollin3: a new marker of squamous differentiation in undifferentiated large-cell carcinoma of the lung, Modern Pathology 22 (2009), 709717.

8. B. Khamar, Desmocollin-3 and Cancer, Biomed J Sci \& Tech Res 5 (2017), 1511-1515.

9. E.P. Salerno, D. Bedognettib, I.S. Mauldin, D.H. Deacon, S.M. Shea, J. Pinczewski, J.M. Obeid, G. Coukos, E. Wang, T.F. Gajewski, F.M. Marincola, C.L. Slingluff Jr. Human melanomas and ovarian cancers overexpressing mechanical barrier molecule genes lack immune signatures and have increased patient mortality risk, Oncoimmunilogy 5 (2016), e1240857.

10.M. Uhlen, P. Oksvold, L. Fagerberg, E. Lundberg, $K$. Jonasson, $M$. Forsberg, M. Zwahlen, C. Kampf, K. Wester, S. Hober, H. Wernerus, L. Björling, F. Ponten, Towards a knowledge-based Human Protein Atlas, Nat Biotechnol. 28 (2010):1248-1250. Human Protein Atlas

https://www.proteinatlas.org/ENSG 
00000134762-DSC3/tissue.

Accessed 03 Feb 2020

11.M. Uhlen, Xi-C. Zhang, S. Lee, E. Sjöstedt, L. Fagerberg, G. Bidkhori, R. Benfeitas, M. Arif, Z. Liu, F. Edfors, K. Sanli, K. Feilitzen, P. Oksvold, E. Lundberg, S. Hober, P. Nilsson, J. Mattsson, J.M. Schwenk, H. Brunnstrom, B. Glimelius, T. Sjoblom, P. Edqvist, D. Djureinovic, P. Micke, C. Lindskog, A. Mardinoglu, F. Pontén, A pathology atlas of the human cancer transcriptome, Science 357 (2017), (eaan2507) 111. Human Protein Atlas https://www.proteinatlas.org/ENSG 00000134762-DSC3/pathology. Accessed 03 Feb 2020

12.A.G. Robertson, J. Kim, H. AlAhmadie J. Bellmunt, G. Guo, A.D. Cherniack, T. Hinove, P.W. Laird, K.A. Hoadley, R. Akbani, M.A.A. Castro, E.A. Gibb, R.S. Kanchi, D.A. Gordenin, S.A. Shukla, F. SanchezVega, D.E. Hansel, B.A. Czerniak, V.E. Reuter, X. SU, B.S. Carvalho, V.S. Chagas, K.L. Mungall, S. Sadeghi, C.S. Pedamallu, Y. LU, L.J. Klimczak, J. Zhang, C. Choo, A.l. Ojesina, S. Bullman, K.M. Leraas, T.M. Lichtenberg, C.J. Wu, N. Schultz, G Getz, M. Meyerson, G.B. Mills, D.J. McConkey, J.N. Weinstein, D.J. Kwiatkowski, S.P. Lerner, Comprehensive Molecular Characterization of Muscle-Invasive Bladder Cancer, Cell 171 (2017), 540-556.

13. Q. Wang, D. Peng, S. Zhu, S. Chen, T. Hu, M. Soutto, R. Saad, S. Zhang, W. El-Rifai, Regulation of Desmocollin3 Expression by
Promoter Hypermethylation is Associated with Advanced Esophageal Adenocarcinomas, Journal of Cancer 5 (2014): 457-464. 14.M.P. Fitzgerald, F. Gourronc, M.L.T. Teoh, M.J. Provenzano, A.J. Case, J.A. Martin, F.E. Domann, Human Chondrosarcoma Cells Acquire an Epithelial-Like Gene Expression Pattern via an Epigenetic Switch: Evidence for MesenchymalEpithelial Transition During Sarcomagenesis, Sarcoma (2011), $1-11$.

15. L. Wang, T. Liu, Y. Wang, L. Cao, M. Nishioka, R.L. Aguirre, A. Ishikawa, L. Geng, N. Okada, Altered expression of desmocollin 3, desmoglein 3, and beta-catenin in oral squamous cell carcinoma: correlation with lymph node metastasis and cell proliferation, Virchows Arch 451 (2011) : 959-966.

16.T. Knösel, Y. Chen, S. Hotovy, U. Settmacher, A. Altendorf-Hofmann, I. Petersen, Loss of desmocollin 1-3 and homeobox genes PITX1 and CDX2 are associated with tumor progression and survival in colorectal carcinoma, Int J Colorectal Dis 27 (2013), 1391-1399.

17. C.P. Belani, B.C. Chakraborty, R.I. Modi, B.M. Khamar, A randomized trial of TLR-2 agonist CADI-05 targeting desmocollin-3 for advanced non-small-cell lung cancer, Annals of Oncology 28 (2017), 298-304.

18.T. Cui, Y. Chen, L. Yang, $M$. Mireskandari, T. Knösel, Q. Zhang, L.H. Kohler, A. Kunze, N. Presselt, I. Petersen, Diagnostic and 
prognostic impact of desmocollins in human lung cancer, J Clin Pathol. 65 (2012) :1100-6.

19.D. Hanahan, R.A. Weinberg, The hallmarks of cancer, Cell 100 (2000), 57-70.

20.D. Hanahan, R.A. Weinberg, Hallmarks of Cancer: The Next Generation, Cell 144 (2011), 646674.

21.M. Iannuccelli, E. Micarelli, P.L. Surdo, A. Palma, L. Perfetto, I. Rozzo, L. Castagnoli, L. Licata, G. Cesareni, Cancer Gene Net: linking driver genes to cancer hallmarks, Nucleic Acids Research 48 (2020) : D416-D421.

22. J.N. Weinstein, E.A. Collisson, G.B. Mills, K.M. Shaw, B.A. Ozenberger, K. Ellrott, I. Shmulevich, C. Sander, J.M. Stuart, The Cancer Genome Atlas Pan-Cancer Analysis Project, Nat Genet. 45 (2013), 1113-1120.

23. J. Gao, B.A. Aksoy, U. Dogrusoz, G. Dresdner, B. Gross, S.O. Sumer, Y. Sun, A. Jacobsen, R. Sinha, E. Larsson, E. Cerami, C. Sander, N. Schultz, Integrative analysis of complex cancer genomics and clinical profiles using the cBioPortal, Sci Signal 6 (2013):1-11.

24. Á. Nagy, A. Lánczky, O. Menyhárt, B. Győrffy, Validation of miRNA prognostic power in hepatocellular carcinoma using expression data of independent datasets, Scientific Reports 8 (2018), 9277.

25. Fire Browse, Broad Institute of MIT and Harvard, (2019), Version: 1.1.40 (2019-10-13

$13: 14: 32$ c66e6f910b6a89397a4de26c). http://firebrowse.org/viewGene.ht
mleggene=DSC3. Accessed 03 Feb 2020

26. Jeremy Stangroom, Social Science Statistics. (Updated 2018) https://www.socscistatistics.com/te sts/spearman/default2.aspx.

(Accessed 03 Feb 2020)

27.S. Babicki, D. Arndt, A. Marcu, Y. Liang, J.R. Grant, A. Maciejewski, D.S. Wishart, Heatmapper: webenabled heat mapping for all, Nucleic Acids Res. 44 (2016), W147W153.

https://doi.org/10.1093/nar/gkw419

Heatmapper

http://heatmapper.ca/expression/. Accessed 03 Feb 2020.

28. M.M. Oshiro, C.J. Kim, R.J. Wozniak, D.J. Junk, J.L. Muñoz-Rodríguez, J.A. Burr, M. Fitzgerald, S.C. Pawar, A.E. Cress, F.E. Domann, B.W. Futscher, Epigenetic silencing of DSC3 is a common event in human breast cancer, Breast Cancer Research 7 (2005), R669-R680.

29. M.H. Bailey, C. Tokheim, E. PortaPardo, S. Sengupta, D. Bertrand, A. Weerasinghe, A.Colaprico, M.C. Wendl, J. Kim, B. Reardon, P.K. Ng, K.J. Jeong, S. Cao, Z. Wang, J. Gao, Q. Gao, F. Wang, E. M. Liu, L. Mularoni, C. Rubio-Perez, N. Nagarajan, I. Cortés-Ciriano, D.C. Zhou, W.W. Liang, J.M. Hess, V.D. Yellapantula, D. Tamborero, A. Gonzalez-Perez, C. Suphavilai, J.Y. Ko, E. Khurana, P.J. Park, E.M. Van Allen, H. Liang, M.S. Lawrence, A. Godzik, N. Lopez-Bigas, J. Stuart, D. Wheeler, G. Getz, K. Chen, A.J. Lazar, G.B. Mills, R. Karchin, L. Ding, Comprehensive Characterization 
of Cancer Driver Genes and Mutations, Cell 173 (2018), 371-385. 30. L.J. Jodi, A.N. Nicole, J.G. Kathleen, Desmosomes: Regulators of cellular signaling and adhesion in epidermal health and disease, Cold Spring Harb Perspect Med 4 (2014), 1-23.

31. M. Berika, D. Garrod, Desmosomal Adhesion In Vivo, Cell Communication \& Adhesion 21 (2014), 65-75.

32.G. Ferone, M.R. Mollo, H.A. Thomason, D. Antonini, H. Zhou, R. Ambrosio, L.D. Rosa, D. Salvatore, S. Getsios, H. Bokhoven, J. Dixon, C. Missero, p63 control of desmosome gene expression and adhesion is compromised in AEC syndrome, Human Molecular Genetics 22 (2013), 531-543.

33.K. Khan, R. Hardy, A. Haq, Desmocollin switching in colorectal cancer, British Journal of Cancer 95 (2006), 1367-1370.

34. K.J. Green, A. Jaiganesh, J.A. Broussard, Desmosomes: Essential contributors to an integrated intercellular junction network, F1000Research, 8 (2019), 2150.

35.R.L. Dusek, L.D. Attardi, Desmosomes: new perpetrators in tumour suppression, Nat Rev Cancer 11 (2011), 317 - 323.

36.M.A. Moses, A.L. George, N. Sakakibara, K. Mahmood, R.M. Ponnamperuma, K.E. King, W.C. Weinberg, Molecular Mechanisms of p63-Mediated Squamous Cancer Pathogenesis, Int. J. Mol. Sci. 20 (2019), 1-21
37.R.A. Ihrie, M.R. Marques, B.T. Nguyen, J.S. Horner, Perp Is a p63Regulated Gene Essential for Epithelial Integrity, Cell 120 (2005), 843-856.

https://doi.org/10.1016/j.cell.2005.0 1.008

38. M.I. Koster, J. Dinella, J. Chen, C. O' Shea, P.J. Koch, Integrating Animal Models and In Vitro Tissue Models to Elucidate the Role of Desmosomal Proteins in Diseases, Cell Commun Adhes. 21 (2014), 55-63

39. K. Srivastava, A. Pickard, S.G. Craig, G.P. Quinn, S.M. Lambe, J.A. James, S.S. McDade, D.J. McCance, $\quad \triangle$ Np63y/SRC/Slug Signaling Axis Promotes Epithelialto-Mesenchymal Transition in Squamous Cancers, Clin Cancer Res 24 (2018) :3917-3927.

40.S.H. Dzinic, M.M. Bernardo, D.S. Oliveira, M. Wahba, W. Sakr, S. Sheng, Tumor suppressor maspin as a modulator of host immune response to cancer, Bosn J of Basic Med Sci 15 (2015), 1-6.

41.T.M. Bodenstine, R.E.B. Seftor, Z. Khalkhali-Ellis, E.A. Seftor, P.A. Pemberton, M.J.C. Hendrix, Maspin: molecular mechanisms and therapeutic implications, Cancer Metastasis Rev 31 (2012), 529-551.

42. J. Lockett, S. Yin, X. Li, Y. Meng, S. Sheng, Tumor suppressive maspin and epithelial homeostasis J. Cell. Biochem. 97 (2005), 651-660.

43.M.A. Nieto, R.Y. Huang, R.A. Jackson, J.P. Thiery, EMT: 2016. Cell 166 (2016), 21-45.

44. J. Pan, Y. Chen, C. Mo, D. Wang, J. Chen, X. Mao, S. Guo, J. Zhuang, S. 
Qiu, Association of DSC3 mRNA Down-Regulation in Prostate Cancer with Promoter
Hypermethylation and Poor Prognosis, PLOS ONE 9 (2014), e92815:1-11. 